\title{
Near field spectroscopy of selectively oxidized vertical cavity surface emitting lasers
}

\author{
J. Kim, J. T. Boyd, ${ }^{\text {a) }}$ and Howard E. Jackson ${ }^{\text {b) }}$ \\ Department of Physics, University of Cincinnati, Cincinnati, OH 4522-0011 \\ K. D. Choquette \\ Photonics Research Department, Sandia National Laboratories, Albuquerque, NM, 87185
}

DEC ? ? 1999

OSTI

\begin{abstract}
Selectively oxidized vertical cavity surface emitting lasers (VCSELs) have been studied by spectrally resolved near field scanning optical microscopy (NSOM). We have obtained spatially and spectrally resolved images of both subthreshold emission and lasing emission from a selectively oxidized VCSEL operating at a wavelength of $850 \mathrm{~nm}$. Below threshold, highly local high gain regions, emitting local intensity maxima within the active area, were observed; these were found to serve as lasing centers just above threshold. Above threshold, the near field spatial modal distributions of low order transverse modes were identified by spectrally analyzing the emission; these were found to be complex and significantly different from those measured in the far field.
\end{abstract}

a) Department of Electrical and Computer Engineering and Computer Science

b) Electronic mail: howard.jackson@uc.edu 
Since the introduction of selectively oxidized vertical cavity surface emitting lasers (VCSELs), ${ }^{1,2}$ significant progress has been achieved in developing high performance VCSELs. Selective oxidation is an efficient and convenient current confinement scheme which, because of superior electrical confinement within the active area, is leading to lower threshold currents. ${ }^{3,4}$ The existence of oxidized layer also provides optical confinement in the laser cavity and thus optical modes are more tightly confined in the active area than for an implanted VCSEL; this results in low diffraction loss enabling high efficiency. ${ }^{4,5}$ The higher optical confinement behavior in oxidized VCSELs also results in more distinct and complex multimode emission, demanding a novel method to identify the VCSEL modal structures. Previously, near field scanning optical microscopy (NSOM) with a subwavelength spatial resolution has been successfully used to identify the spatial and spectral mode structures of proton implanted VCSELs. ${ }^{6-8}$ We report here on spectrally-resolved NSOM results which show directly that the oxidized VCSEL has a significantly different modal distribution than an implanted VCSEL, both in subthreshold emission and lasing emission. Furthermore below threshold local high gain regions, emitting local intensity maxima within the active area, were found to serve as lasing centers just above threshold. Above threshold the near field spatial modal distributions of low order transverse modes were identified by spectrally analyzing the emission; these were found to be complex and significantly different from that measured in the far field.

A selectively oxidized VCSEL having a $5 \mathrm{~m}$ square oxide aperture was investigated. In the top and bottom AlGaAs mirrors, $40 \mathrm{~nm}$ layers (approximately quarter wavelength thick) were oxidized in the third period above and below the active region which contained five $8 \mathrm{~nm}$ quantum wells. The emission wavelength was around $850 \mathrm{~nm}$ which 


\section{DISCLAIMER}

This report was prepared as an account of work sponsored by an agency of the United States Government. Neither the United States Government nor any agency thereof, nor any of their employees, make any warranty, express or implied, or assumes any legal liability or responsibility for the accuracy, completeness, or usefulness of any information, apparatus, product, or process disclosed, or represents that its use would not infringe privately owned rights. Reference herein to any specific commercial product, process, or service by trade name, trademark, manufacturer, or otherwise does not necessarily constitute or imply its endorsement, recommendation, or favoring by the United States Government or any agency thereof. The views and opinions of authors expressed herein do not necessarily. state or reflect those of the United States Government or any agency thereof. 


\section{DISCLAIMER}

Portions of this document may be illegible in electronic image products. Images are produced from the best available original document. 
red-shifted with increasing injection current due to the temperature dependence of the refractive indices. The NSOM collection mode has been used here, where a tip with a $100 \mathrm{~nm}$ optical aperture is placed above the laser output aperture thus collecting the laser emission from the sample mounted on a piezo stage. The regulation of constant tip height in the near field $(<20 \mathrm{~nm}$ ) was achieved using shear force detection. For far field measurements in various heights from the laser surface, the sample stage was moved vertically with respect to the NSOM tip. To obtain the emission patterns of the samples without any spectral information, collected light was counted with a photon counting system. For spectroscopic NSOM measurements, collected light from the tip is coupled into a triple spectrometer and the full spectra (spectral range $\sim 2 \mathrm{~nm}$ ) were recorded by a nitrogen cooled CCD at each pixel position. (WHAT WAS SPECTRAL RESOLUTION?) A typical scan area was $7 \times 7 \mathrm{~m}$ with $0.2 \mathrm{~m}$ step size with a 1 second acquisition time.

In Fig. 1, the near field emission pattern at $1.5 \mathrm{~mA}$ injection current (subthreshold) is shown. Subthreshold emission can provide direct information on the lateral gain profile of the active area since it is spontaneous emission from the quantum wells in the active region. ${ }^{9,10}$ Thus one sees a square-like emission pattern indicative of the shape of the active area defined by lateral oxidation. Note the emission intensity profile is rather flat across the aperture in Fig. 1(a). The plateau profile of subthreshold emission indicates overall uniform lateral current injection into the active area. This is in contrast to the case of implanted VCSELs which show a Gaussian-like emission profile at subthreshold injection currents. ${ }^{8}$ Unlike the boundary of ion implanted VCSEL active area, the boundary of an oxide VCSEL active area is very distinct, suggesting that the 
electrical confinement of injected carriers into the active area achieved by oxide apertures is superior to the implanted VCSELs. ${ }^{11}$ Note also the detailed structures on the plateau of the subthreshold emission displayed in Fig. 1a. As the contour plot in Fig lb shows, the upper region and the left side region (indicated with arrows) each have a somewhat higher emission. This suggests the existence of a higher density of injected carriers in these regions; since these regions will experience higher gain (provided by the higher carrier densities), lasing action should occur preferentially in these regions.

A lasing threshold at $2.2 \mathrm{~mA}$ and a linear increase output power with injection current were observed from the sample. As the current goes over the lasing threshold, the broad plateau emission profile changes into several localized emissions. As shown in Fig. 2a, just above the threshold at $3.0 \mathrm{~mA}$, the emission pattern consists of several local maximum intensity regions. An even more complex emission pattern is observed in Fig. $2 \mathrm{~b}$ at a higher current of $6.5 \mathrm{~mA}$. The same regions (indicated with arrows) which showed slightly higher emission in subthreshold emission now serve as distinct lasing centers. The upper region, which had the highest intensity in the subthreshold emission, lases first as shown in Fig. 2(a), and subsequently the region on the left side region lases at the higher injection current, as shown in Fig. 2(b). Thus, certain regions within the laser cavity are regions preferred for carrier injection creating locally higher gains and thus local intensity maxima in these regions.

We have observed a number of transverse modes at all injection currents above lasing threshold. To obtain modal distributions of individual transverse modes, we have spectrally analyzed the laser emissions. Figures 3a displays representative spectra in the 
near field spatial distributions of the fundamental mode and one of the first higher order modes.

The spatial distribution of the fundamental mode (Fig. 3a) at a wavelength of $852.04 \mathrm{~nm}$ displays a circular shape located near the aperture center with a $2.5 \mathrm{~m}$ full width. The first order modes at $851.42 \mathrm{~nm}$ have a double lobe shape, similar to the shape observed from the $\mathrm{LP}_{11}$ mode of implanted VCSELs. ${ }^{7,8}$ These modal patterns of fundamental and the first order transverse modes obtained in the near field have local intensity maxima as has been discussed above. Note that each of these figures is a single mode emission pattern, indicating that the complex emission pattern is not due to the presence of multiple modes.

To investigate the propagation of these individual transverse modes away from the VCSEL surface, measurements of the spatial distribution of modes were carried out $10 \mathrm{~m}$ away from the laser surface, i.e. in the far field. Figure 3(b) display emission patterns, now measured in the far field at $10 \mathrm{~m}$ away from the laser surface. A smoother distribution for both fundamental and the first order mode is observed with no evidence of the local intensity maxima observed in the near field pattern. We attribute this loss of detail of the emission structures to the lower spatial resolution of the emission pattern obtained in the far field. We found that the far field pattern of the fundamental mode has the same width as the one obtained in the near field and the distance between the two lobes of the first order mode is the same as measured in the near field

In summary we have reported a spectrally resolved NSOM investigation on a selectively oxidized VCSEL. Using NSOM, we have obtained images of subthreshold emission and lasing emission from the VCSEL. We have identified local high gain 
regions emitting local intensity maxima below threshold; as the current goes above threshold, these same regions are found to serve as lasing centers. The spatial modal distributions of individual transverse modes were identified by spectrally analyzing the emission with high spectral resolution in both the near field and far field. The near field or NSOM data provided unique spatially local information on the emission characteristics of the selectively oxidized vertical cavity surface emitting laser.

The work at University of Cincinnati was supported in part by ARO DAAG55-97-10378 and NSF ECS-9412772. The work at Sandia National Laboratories is supported by the United States Department of Energy under Contract DE-AC04-94AL85000. 


\section{References}

[1] D. L. Huffaker, D. G. Deppe, K. Kumar, and T. J. Rogers, Appl. Phys. Lett. 65, 97 (1994).

[2] K. D. Choquette, R. P. Schneider Jr., S. P. Kilcoyne, and K. M. Geib, Electron. Lett. 302043 (1994).

[3] G. M. Yang, M. H. MacDougal, and P. D. Dapkus, Electron. Lett. 31, 886 (1995).

[4] K.L. Lear, K.D. Choquette, R.P. Schneider, Jr., S.P. Kilcoyne, and K.M. Geib, Electron. Lett. 31,.208 (1995).

[5] K.L. Lear, R.P. Schneider, K.D. Choquette, Jr., S.P. Kilcoyne, and K.M. Geib, IEEE Photon. Tech. Lett. 8, 740 (1996).

[6] I. Horsch, R. Kusche, O. Marti, B. Weigl, and K.J. Ebeling, J. Appl. Phys. 79, 3831 (1996).

[7] J. Kim, D. E. Pride, J. T. Boyd, and H. E. Jackson, Appl. Phys. Lett. 71, 2616 (1998).

[8] J. Kim, D. E. Pride, J. T. Boyd, and H. E. Jackson, Proceedings of the $24^{\text {th }}$ International Conference on the Physics of Semiconductors, ed. D. Gershoni, World Scientific Press, London,(1999).

[9] G.C. Wilson, D.M. Kuchta, J.D. Walker and J.S. Smith, Appl. Phy. Lett. 64, 542 (1994).

[10] L. Raddatz, I. H. White, H. D. Summers, K. H. Hahn, M. R. Tan, and S. Y. Wang, IEEE Photon. Technol. Lett. 8743 (1996) 
[11] K. D. Choquette, K.L. Lear, R. P. Schneider Jr, and K. M. Geib, Appl. Phy. Lett. 66, 3413 (1995). 


\section{Figure captions}

Figure 1 A near field profile of subthreshold emission of an oxidized VCSEL obtained at a current of $1.5 \mathrm{~mA}$. Fig. 1a is a false color contour plot with red representing the most intense emission. Figure $1 \mathrm{~b}$ is a $3 \mathrm{D}$ representation of the same data. Note in each case the local high emission regions indicated with arrows.

Figure 2 Lasing emission patterns obtained at $3.0 \mathrm{~mA}$ (Fig. 2a) and $6.5 \mathrm{~mA}$ (Fig. 2b). Just above the threshold current lasing is initiated from the region where higher subthreshold emission were observed (indicated with the upper arrow). Additional local emissions are observed at a higher injection current of $6.5 \mathrm{~mA}$, including the lower left region (indicated with an arrow).

Figure 3 Spatial modal distribution of a fundamental mode and a first order transverse mode obtained in the near field (Fig. 3a) and $10 \mathrm{~m}$ way from the laser surface (Fig. 3b). The fundamental mode displays a circular shape located on the aperture center and the first order transverse mode displays a double lobe shape similar to $\mathrm{LP}_{11}$ mode observed from implanted VCSELs. Note this single wavelength emission displayed in Fig. 3a consists of several local intensity peaks. Due to the lower spatial resolution, a smooth emission pattern is observed in the far field (Fig. 3b). 

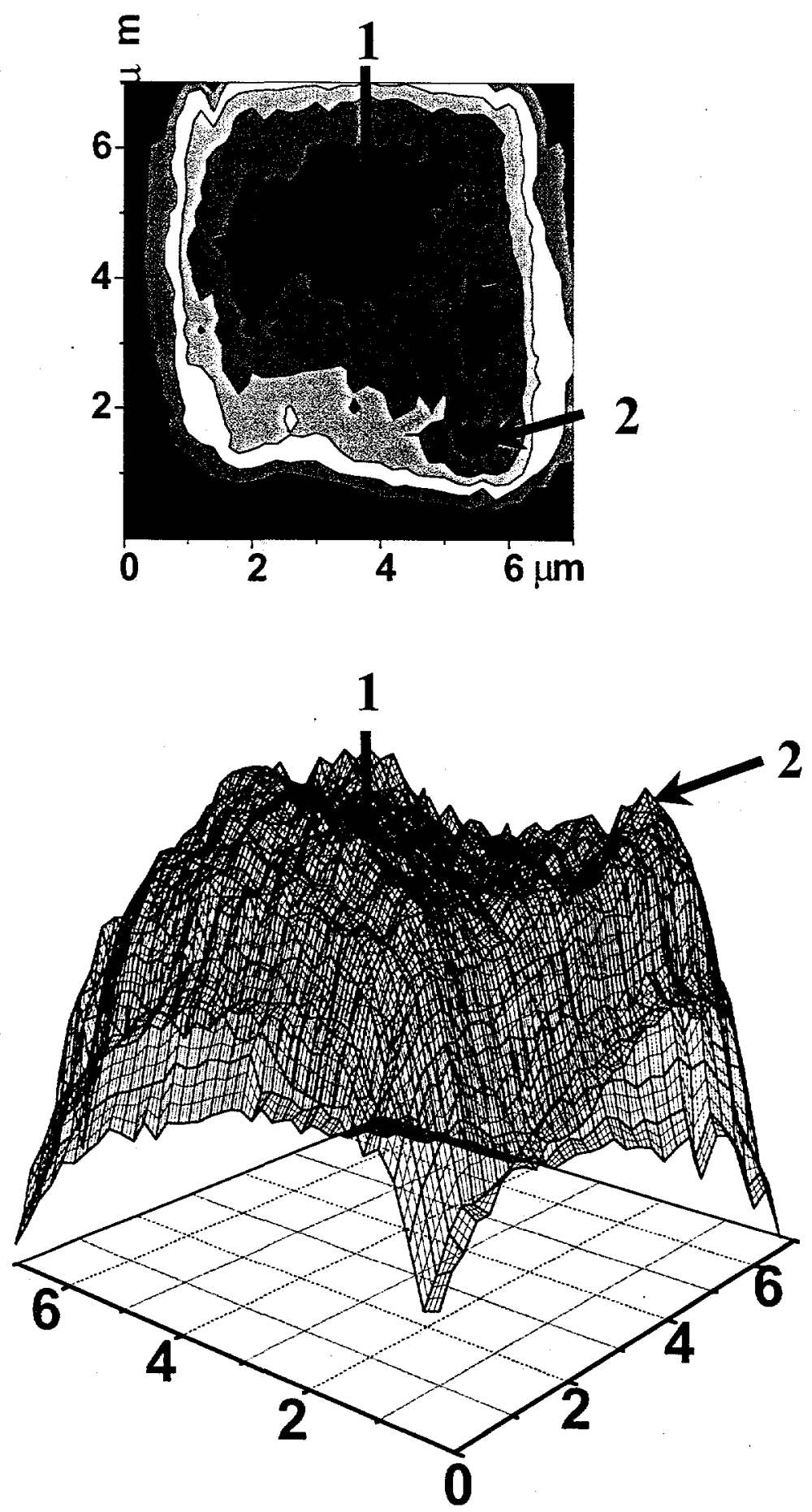

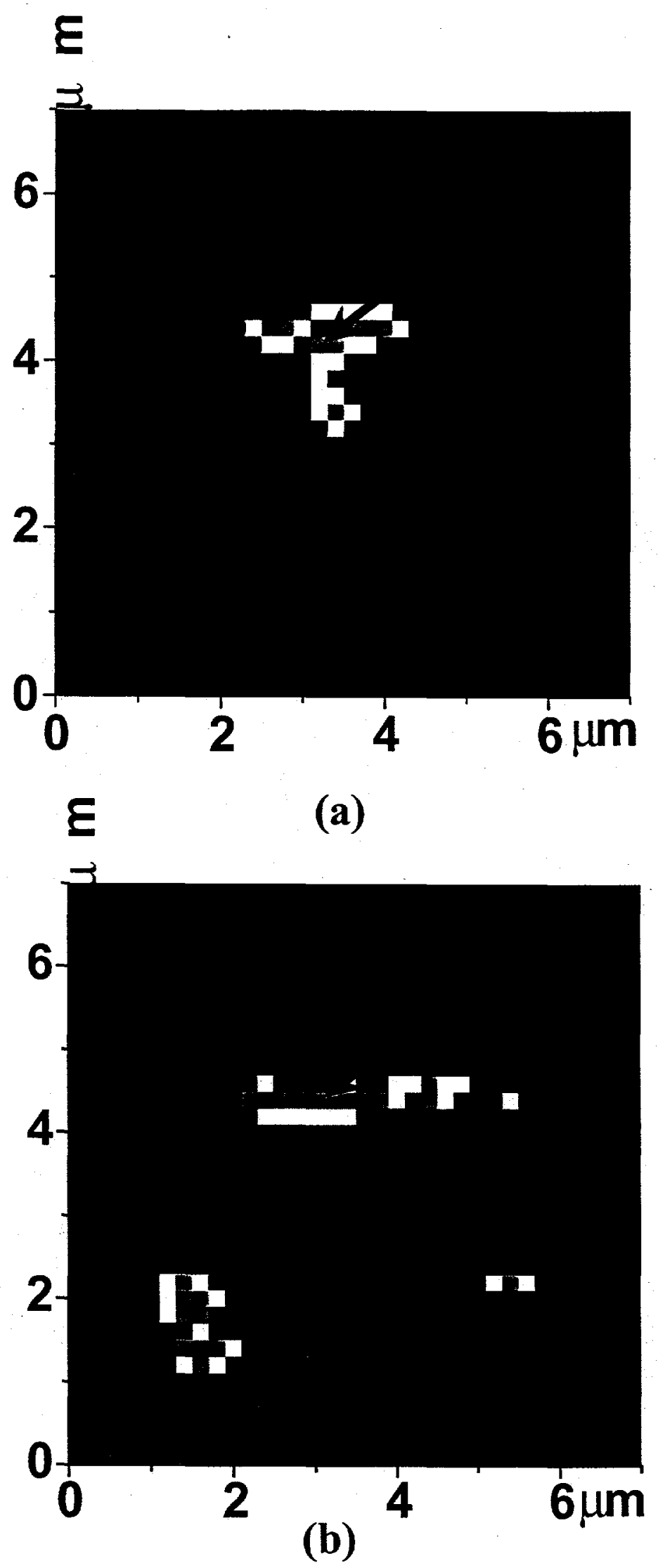

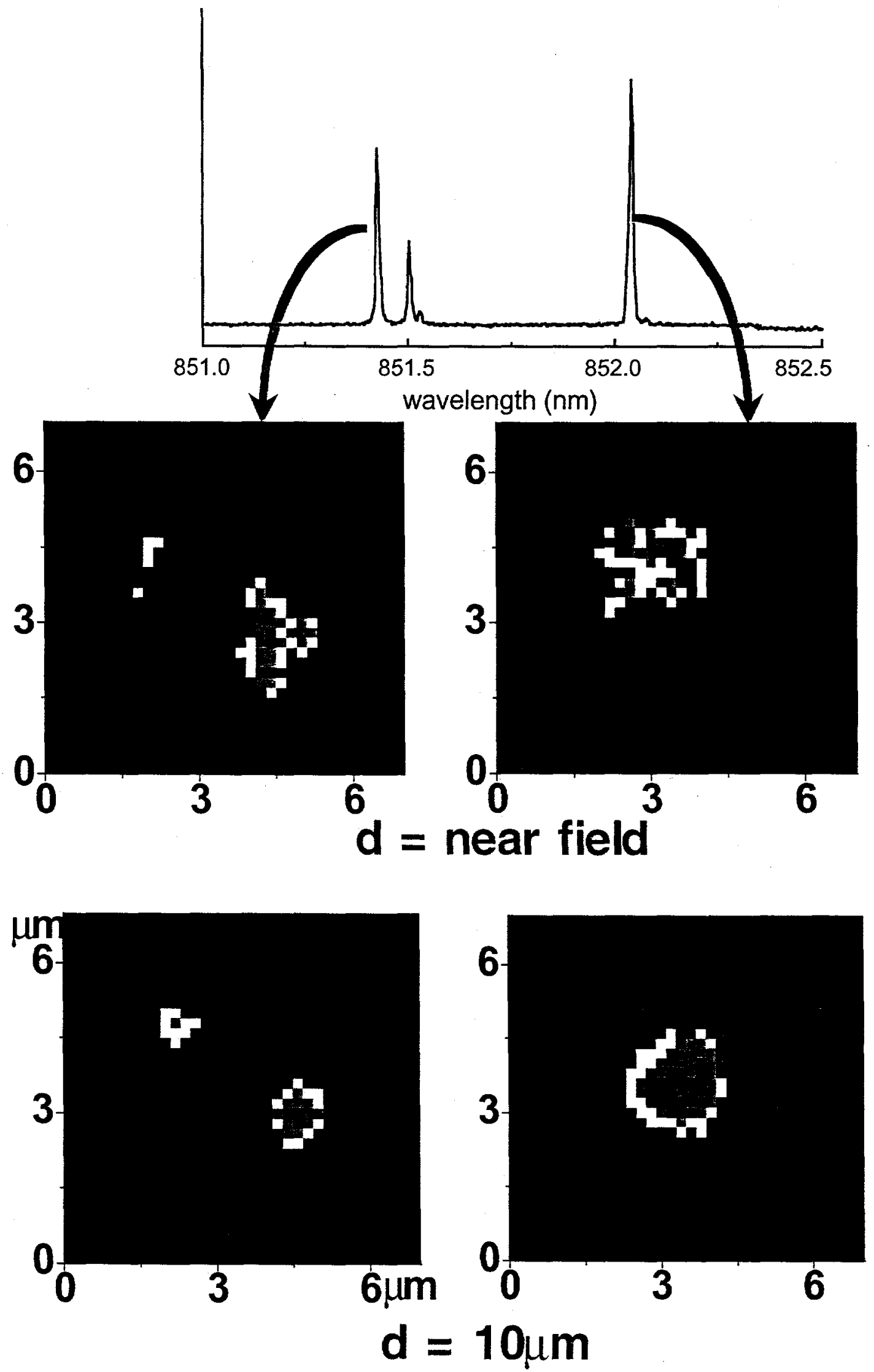